\title{
Thermal Modelling Approach and Model Predictive Control of a Water-cooled PEM Fuel Cell System
}

\author{
J.D. Rojas, Member, IEEE, \\ Universidad de Costa Rica \\ San José, Costa Rica \\ email: jdrojas@eie.ucr.ac.cr
}

\author{
C. Ocampo-Martinez, Member, IEEE, \\ Inst. de Robòtica i Informàtica Industrial \\ (CSIC-UPC) Barcelona, Spain \\ email: cocampo@iri.upc.edu
}

\author{
C. Kunusch, Member, IEEE, \\ Inst. de Robòtica i Informàtica Industrial \\ (CSIC-UPC) Barcelona, Spain \\ email: ckunusch@iri.upc.edu
}

\begin{abstract}
In this paper, a modular thermal modelling approach for a water-cooled Proton Exchange Membrane fuel cell (PEM-FC) stack is proposed. The resultant model is based on the heat flux inside the stack and has been validated in a real test station. Then, a linear model predictive control (MPC) strategy for temperature regulation is designed and tested in simulation with promising results. The proposed MPC controller not only tackles the temperature regulation problem, but also incorporates necessary management constraints for the proper operation of the system in real conditions.
\end{abstract}

\section{INTRODUCTION}

Fuel cells are devices that convert the energy of a chemical reaction directly into electricity without a combustion process [1], [2]. The fuel in general can be considered to be hydrogen that, combined with oxygen, produces heat, water and electricity. The operating principle was first discovered by William Grove in 1893 , but is until the mid of the 20th century that fuel cells have become a viable technology and a promising alternative to combustion engines [1].

The proper modelling of the temperature variation is relevant, not only from a scientific point of view, but also as an industrial need. Several modelling methodologies have been presented in the literature for Proton Exchange Membrane fuel cells (PEM-FC) with water cooling systems. In [3] a nodal network approach is used to model the thermal behaviour of a $150 \mathrm{~W}$ PEM-FC. In [4], both the sensible and the latent heat are taken into account to model the thermal dynamics of a Ballard PEM-FC stack. In [5], a 3-D nodal modelling approach is used and implemented using parallel computing in order to have an accurate result by dividing the stack in several nodes.

The problem with these models is that they are either quite simple and only consider the PEM-FC as a single mass with the same temperature or they are too complex and, therefore, require higher computational burden. The modelling approach presented in this work is a compromise: simple but meaningful at the same time. It is based on a modular approach oriented to control applications and useful to predict the temperature of each cell in a stack, taking into account the heat flow going from every cell to the cooling water.

From a control point of view, a fuel cell device can be depicted as a multi-variable system in which certain hard constraints have to be taken into account for its proper operation.
As any real system, fuel cells are plenty of behaviours and variables bounded by physical limits, e.g., ranges of voltages, currents, flows, which should be considered when a selected control law is designed. Moreover, the interaction of the diverse compositional sub-systems determines the definition of several operational constraints that, in the same way as the variable bounds, should be taken into account when formulating a closed-loop control scheme. In the case of PEMFC stacks, the temperature regulation is relevant due to the fact that it affects its efficiency. Moreover, this regulation and the associated temperature constraints in the coolant device are related to the overall system durability.

The consideration of model predictive control (MPC) strategies in dynamical systems such as PEM-FCs is nowadays an incipient topic in the literature. However, the use of those control strategies is becoming more widespread due to the particular capabilities of the technique, which allows solving crucial problems related to the control and management of the fuel cell dynamics. In this sense, MPC has been recognized as a more powerful methodology than other control schemes since it has the intrinsic ability to deal with system constraints in a systematic and straightforward manner [6].

Added to this fact, there exist other strong reasons for utilizing this control technique apart of the constraints handling such as the capability of considering several variables (multivariable systems) and control objectives (multi-objective control) as well as the consideration of system disturbances in on-line mode.

MPC is very sensitive to the model accuracy since the control computation is precisely based on a mathematical model of the plant. This dependence opens several ways for the design of the MPC controllers according to the nature of the fuel cell model: from the purely non-linear MPC [7], to linear approaches [8], [9], piece-wise affine (PWA) models [10], [11] and hybrid systems forms [12]. Regarding the incorporation of actuator limitations and state constraints in the controller design, [13] discusses those aspects for the prevention of fuel cell starvation within the MPC framework.

In this paper, the design of a linear MPC approach with off-set free characteristics is presented in order to control the temperature of a PEM-FC system while considering relevant operational constraints over the variables of the model. The MPC controller design is based in the linearisation of an 


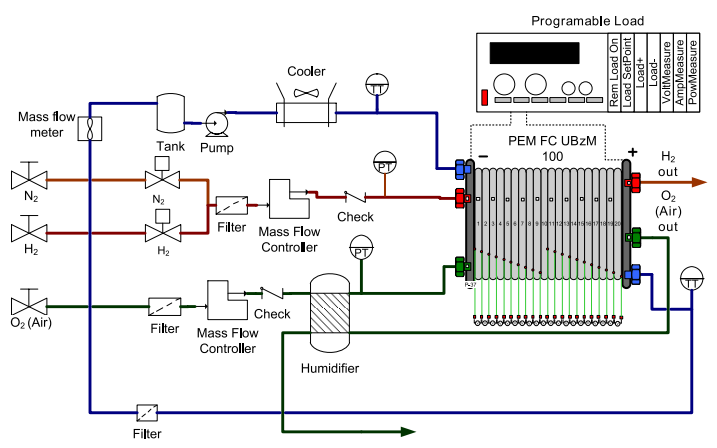

Fig. 1. Layout of the Fuel Cell Station. TT stands for Temperature transducer and PT for pressure transducer.

accurate non-linear thermal model for the FC system. Both resultant models (of linear and non-linear nature, respectively) have been validated on a real PEM-FC test station. The main contribution of this paper is twofold. First, a thermal modelling methodology for water cooled PEM-FC stacks is proposed, explained and discussed. Second, an MPC controller based on the resultant model and with null steady-state error is designed and tested in simulation.

The remainder of this paper is organised as follows. The PEM-FC test station and the thermal model are presented in Section II. The proposed MPC design is shown in Section III, while the discussion of the main results is presented in Section IV. Finally, the conclusions are drawn in Section V.

\section{Thermal Modelling OF THE PEM-FC STATION}

A diagram of the station is presented in Fig. 1. The stack is a $600 \mathrm{~W}$ PEM-FC, model BZ100 manufactured by $\mathrm{UBzM}^{\circledR}$. It has 20 cells with $100 \mathrm{~cm}^{2}$ of active area and the opencircuit voltage is approximately $0.95 \mathrm{~V}_{\mathrm{DC}} /$ cell. The maximum current is $50 \mathrm{~A}$ at a nominal operating voltage of $12 \mathrm{~V}$. The fuel cell stack is cooled using demineralised water through special channels connected in parallel between each cell. This cooling system is composed by a water flow meter, a small deposit tank, an electric water pump and a heat exchanger with two fans for forced convection. It has also temperature transducers in both the inlet and the outlet of the PEM-FC.

In Fig. 2, the general block diagram of the model is depicted, where $V$ is the voltage of the complete stack, $I$ is the stack current, $T_{a m b}$ is the room temperature, $D_{\text {pwm }}$ is the duty cycle that defines the velocity of the fans of the cooling system, $T_{r c}$ is the temperature of the heat exchanger walls, $T_{\text {woutc }}$ is the temperature of the output water of the cooling system, which is considered to be equal to $T_{\text {wins }}$, the input water temperature of the stack, $T_{\text {winc }}$ in the input water temperature to the cooling system, which is also considered to be equal to $T_{\text {wouts }}$. All inputs are considered as measurable disturbances, except $D_{\text {pwm }}$, which is treated as a manipulated input (control action).

\section{A. Thermal Modelling of the Stack}

In [14], a general thermal modelling approach for a fuel cell is made following the connections between each different

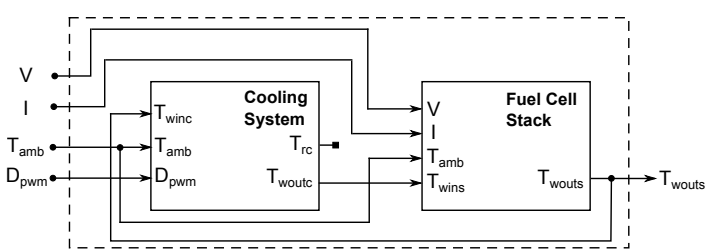

Fig. 2. Block Diagram of the thermal model of the station.

layer in the cell: the steel end-plates, the graphite plates, the synthetic joints, the anode channels, the membrane, the gases collectors and the cathode channels. The heat flows are analysed for each section and an analogous electrical circuit is used to represent the dynamic behaviour of the system. The model of every single cell can be replicated in order to have the complete thermal model of the stack. In the case of [14], the steel plates have external heating and the fuel-cell stack does not have any cooling system. For the stack of the station under study, it is necessary to restructure all the equations in order to represent the heat flow by convection in the cooling circuit. According to [15], the heat flow from the wall to a fluid is principally by conduction, since there is a viscous layer close to the wall where the gradient of temperature is greater than in the rest of the fluid. Then, the average temperature of the fluid would be lower than the value of this viscous layer.

This heat in the fuel cell is removed by the circulating water, producing a temperature gradient between the input and the output of the coolant line, which is given by ${ }^{1}$

$$
Q_{c}=\dot{m} c_{p}\left(T_{c, \text { out }}-T_{c, \text { in }}\right),
$$

where $Q_{c}$ is the heat removed from the cell, $c_{p}$ is the specific heat capacity of the water, $\dot{m}$ is the water flow (which is maintained constant in the station for security reasons) and $T_{c, \text { out }}$ and $T_{c, \text { in }}$ are the output and input water temperatures, respectively. In the sequel, all temperatures are given in $\mathrm{K}$.

The model that incorporates the heat flow to the refrigerant is depicted in Fig. 3. The parameters of the model are

- $R_{i}$, with $i \in\{1, \ldots, 9\}$, are the respective equivalent thermal resistance between sections, given in $\mathrm{K} / \mathrm{W}$.

- $C_{a i}, C_{b a i}, C_{b}, C_{b a d}, C_{a d}$ and $C_{c p}$ are respectively the thermal capacitance of each section, given in $\mathrm{J} / \mathrm{K}$.

- $\frac{1}{h A}$ is the equivalent thermal resistance between the cathode and the cooling fluid, where $h$ is the heat transfer coefficient given in $\mathrm{W} /\left(\mathrm{m}^{2} \mathrm{~K}\right)$ and $A$ is the effective area of the water channel given in $\mathrm{m}^{2}$.

- $\frac{1}{\dot{m} C_{p}}$ is the equivalent thermal resistance for the gradient in the coolant.

On the other hand, the model variables are

- $T_{a m b}$ is the room temperature, $T_{c i n}$ is the temperature of the coolant at the input, $T_{\text {cout }}$ is the temperature of the coolant at the output, $T_{c p}$ is the temperature of the coolant plate, $T_{b c}$ is the temperature in the graphite plate of the cathode side of the membrane and $T_{b a}$ is the temperature

${ }^{1}$ From now on, the time dependence of the system variables is intentionally omitted for clarity and space restrictions. 


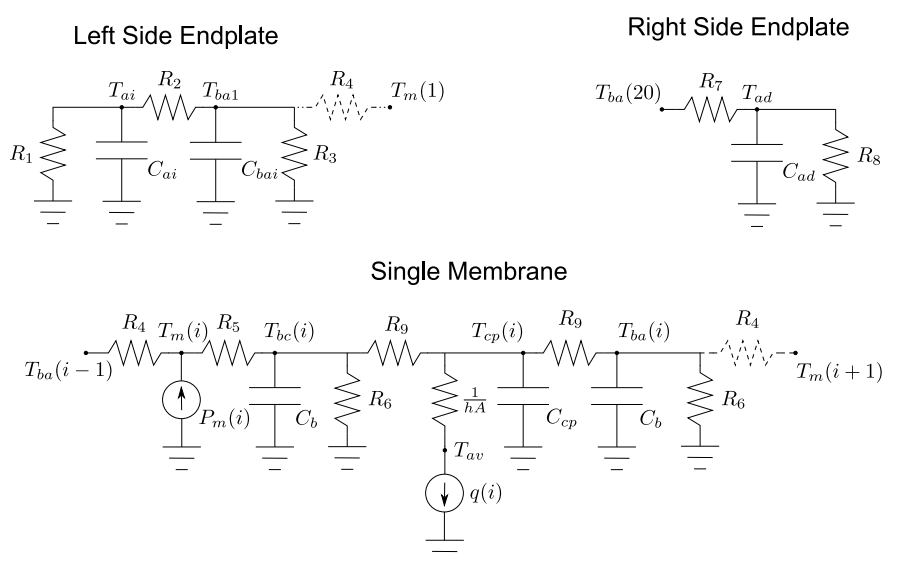

Fig. 3. Submodels of the complete PEMFC.

of the graphite plate in the anode side of each membrane and finally $T_{m}$ is the temperature of the membrane.

- $P_{m, i}$ is the power dissipated as heat by the electrochemical reaction for each membrane, which is related to the voltage of each cell $v_{c, i}$ and the current of the stack $I$.

Analysing the stack structure, it is considered that the cooling circuit is placed between the anode of one cell and the cathode on the next. Therefore, it is assumed that the temperature of the water at the input of each cell is the same as the inlet water temperature of the stack. The average of the outlet water temperatures for all cells is used as an approximation for the outlet water temperature.

The thermal model is divided into three main sections: (i) the left-side plate submodel, which takes into account the thermal capacity of the aluminium left-side plate and the first thermal capacity of the graphite plate; (ii) the membrane submodel, which considers the heat generated by the cathode reaction, as well as the water cooling effects ${ }^{2}$; and (iii) the right-side plate submodel, which is similar to the left-side plate. In Fig. 3, the diagram of each submodel is presented. For space restrictions, only the equations regarding the membrane subsection are presented in this paper. The rest of the equation can be derived by following the heat flow between sections. The generic equations for the membranes are given by (for $i=1,2, \ldots, 20)$

$$
\begin{aligned}
& \frac{d T_{b c, i}}{d t}=\frac{1}{C_{b}}\left[-\left(\frac{1}{R_{5}}+\alpha_{1}\right) T_{b c, i}+\frac{1}{R_{5}} T_{m, i}+\alpha_{2}\right] \\
& \frac{d T_{b a, i}}{d t}=\frac{1}{C_{b}}\left[-\left(\frac{1}{R_{4}}+\alpha_{1}\right) T_{b a, i}+\frac{1}{R_{4}} T_{m, i+1}+\alpha_{2}\right]
\end{aligned}
$$

$$
\frac{d T_{c p, i}}{d t}=\frac{1}{C_{c p}}\left[-\left(\frac{2}{R_{9}}\right) T_{c p, i}+\frac{1}{R_{9}} T_{b c, i}+\frac{1}{R_{9}} T_{b a, i}-q_{i}\right],
$$$$
T_{\text {cout }, i}=2 h A \gamma T_{c p, i}+\left(2 \dot{m} c_{p}-h A\right) \gamma T_{c i n}
$$

${ }^{2}$ This section must be replicated 20 times to take into account all the cells of the particular PEM-FC stack.

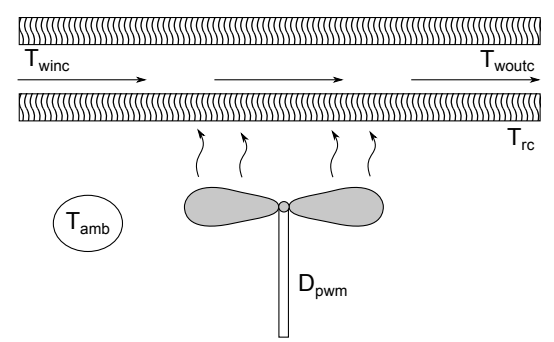

Fig. 4. Simplified diagram of the cooling system

$$
\begin{aligned}
q_{i} & =2 h A \dot{m} c_{p} \gamma\left(T_{c p, i}-T_{c i n}\right), \\
T_{m, i} & =\frac{\lambda}{R_{4}} T_{b a, i-1}+\frac{\lambda}{R_{5}} T_{b c, i}+\lambda P_{m, i}, \\
P_{m, i} & =\left(1.254-v_{c, i}\right) I
\end{aligned}
$$

where $\alpha_{1}=\frac{1}{R_{6}}+\frac{1}{R_{9}}, \alpha_{2}=\frac{1}{R_{9}} T_{c p, i}+\frac{1}{R_{6}} T_{a m b}, \gamma=\frac{1}{h A+2 \dot{m} c_{p}}$, $\lambda=\frac{R_{4} R_{5}}{R_{4}+R_{5}}, v_{c, i}$ is the voltage of the $i$-th cell and $I$ is the current drawn from the stack. The model has been validated using real data, given less than $1 \%$ error in steady state and suitable predictions for transient state in all the operation range.

\section{B. Thermal Modelling of the Cooling System}

The conceptual diagram of the cooling system is depicted in Fig. 4. The velocity of the fans is manipulated with a phase width modulated (PWM) signal with duty cycle $D_{\text {pwm }}$. In this case, a gray-box modelling approach is chosen. This modelling lets the user calibrate the parameters of a model based on physical principles by using an optimization procedure [16]. In this paper, the importance of the cooling system model is to reproduce the dynamic variation of the water input temperature of the stack as a function of the PWM signal of the fans.

The proposed equations of the cooling system model are written as follows:

$$
\begin{aligned}
\frac{d T_{r c}}{d t} & =K_{1} D_{\text {pwm }}\left(T_{a m b}-T_{r c}\right)+K_{2}\left(T_{\text {woutc }}-T_{r c}\right) \\
& +K_{3}\left(T_{a m b}-T_{r c}\right), \\
\frac{d T_{\text {woutc }}}{d t} & =K_{4}\left(T_{r c}-T_{\text {woutc }}\right)+K_{5}\left(T_{\text {winc }}-T_{\text {woutc }}\right),
\end{aligned}
$$

where $D_{\text {pwm }}$ is considered as an input to the system, $T_{r} c$ is the temperature of the heat exchanger walls (considering them as a lumped mass) and $T_{\text {woutc }}$ are the input and output water temperature, respectively. $K_{i}, i \in\{1, \ldots, 5\}$, are the model parameters that were calibrated to develop the model. The water is cooled by the air flow from the fans. Depending on the velocity of the air (and therefore, depending on $D_{\text {pwm }}$ ), the heat flow may vary. In (4), a simple proportional variation is considered. The other components correspond to the energy flow due to different gradients of temperatures (between the water and the walls of the heat exchanger, the walls and the air, and between the input and output water temperature). 


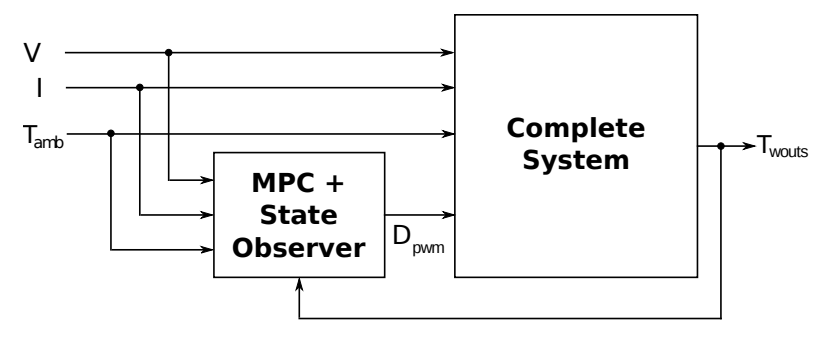

Fig. 5. Proposed control scheme for the station.

\section{MPC CONTROLler DESIGN AND Simulation}

The considered control scheme is depicted in Fig. 5. The controlled variable is the output water temperature of the stack, denoted by $T_{\text {wouts }}$, and the control action is $D_{\text {pwm }}$. For the specific case of the stack and the cooling system, a constraint has to be incorporated in order to maintain the temperature difference between the water inflow and outflow below certain value. The reason behind this restriction is to avoid undesirable condensation inside the stack due to the different temperatures across the stack.

The MPC methodology selected in this paper is based on linear, low-order, discrete time model of the station. Therefore, it is necessary to adjust the complete model presented in Sections II-A and II-B. For the case of the thermal model, if the stack power is considered as a input, this sub-model becomes linear and just an order reduction step is necessary. The procedure to find a reduced order model is first to balance the state space realization, and then discard the states associated with the smaller singular values. For the cooling system, a linearisation around a given operation point was performed. The complete model was discretised using a zeroorder hold equivalent. Hence, an eight-state model was chosen as an approximation of the non-linear model. Using this linear model, a full-state Luenberger observer is also implemented to obtain an estimation of the states [17].

In order to design the MPC controller, consider the discretetime state-space linear model

$$
\begin{aligned}
x(k+1) & =A x(k)+B u(k)+E v(k), \\
y(k) & =C x(k)+D u(k)+F v(k),
\end{aligned}
$$

where $x(k) \in \mathbb{R}^{n}$ is the vector of the system states, $u(k) \in$ $\mathbb{R}^{m}$ is the vector of control signals, $y(k) \in \mathbb{R}^{p}$ is the vector of system outputs, $v(k) \in \mathbb{R}^{q}$ is the vector of the measured disturbances and $k \in \mathbb{Z}_{+}$denotes the discrete time. Moreover, $A, B, C, D, E$, and $F$ are the system matrices of suitable dimensions. Moreover, let $^{3}$

$$
\boldsymbol{\Delta} \mathbf{u}(x(k)) \triangleq\left(\Delta u(0 \mid k), \Delta u(1 \mid k), \ldots, \Delta u\left(H_{p}-1 \mid k\right)\right),
$$

be the sequence of input slew rates defined by

$$
\Delta u(k) \triangleq u(k)-u(k-1)
$$

\footnotetext{
${ }^{3}$ As notation, $z(k+i \mid k)$ denotes the prediction of the variable $z$ at time $k+i$ performed at $k$. For instance, $x(k+i \mid k)$ denotes the prediction of the state, starting from $x(0 \mid k)=x(k)$
}

over a fixed-time prediction horizon $H_{p}$. Notice that (6) depends on the initial state $x(0 \mid k) \triangleq x(k)$, where $x(0)$ is the measured or estimated current state (initial condition). Hence, an MPC controller design is based on the solution of the openloop optimisation problem (OOP)

$$
\begin{gathered}
\min _{\Delta \mathbf{u}} J(x(0), \mathbf{\Delta} \mathbf{u})=\sum_{i=0}^{H_{p}-1}\left[\|y(k+i \mid k)-r(k \mid k)\|_{W_{y}}^{2}\right. \\
\left.+\|\Delta u(k+i \mid k)\|_{W_{r}}^{2}\right],
\end{gathered}
$$

subject to

$$
\begin{aligned}
& x(i+1 \mid k)=A x(i \mid k)+B u(i \mid k)+E v(i \mid k), \\
& y(i \mid k)=C x(i \mid k)+D u(i \mid k)+F v(i \mid k), \\
& G_{1} x(i \mid k)+G_{2} u(i \mid k) \leq g,
\end{aligned}
$$

for all $i \in\left[0, H_{p}-1\right]$, where $J(\cdot): \mathbb{R}^{(m+p) H_{p}} \mapsto \mathbb{R}$ in (8a) is the cost function, $H_{p}$ denotes the prediction horizon or output horizon, $r(k)$ is a vector containing the output references, and $G_{1}, G_{2}$ and $g$ are matrices with suitable dimensions. $W_{r}$ and $W_{y}$ are the weighting matrices that determine the prioritisation of the control objectives. Expression (8d) collects the system constraints for states and control inputs. These constraints are of physical nature and represent the bounds for each one, i.e., $x \in\left[x^{\min }, x^{\max }\right]$ and $u \in\left[u^{\min }, u^{\max }\right]$. Besides, (8d) also includes the system constraint

$$
T_{\text {diff }} \leq T_{\text {diff }}^{\max },
$$

where $T_{\text {diff }}=T_{\text {wins }}-T_{\text {wouts }}$. In this particular case, $T_{\text {diff }}^{\max }=$ $10 \mathrm{~K}$. Assuming that the OOP $(8)$ is feasible, i.e., $\boldsymbol{\Delta} \mathbf{u}(x(k)) \neq$ $\emptyset$, there is an optimal solution given by the sequence of control inputs (obtained by using (7))

$$
\mathbf{u}(k)^{*} \triangleq\left(u(0 \mid k)^{*}, u(1 \mid k)^{*}, \ldots, u\left(H_{p}-1 \mid k\right)^{*}\right),
$$

and then the receding horizon philosophy sets $u_{\mathrm{MPC}}(x(k)) \triangleq u^{*}(0 \mid k)$, with the whole process repeated at the next time instant $k \in \mathbb{Z}_{+}$.

In order to include the offset-free feature in the controller design, it is necessary to perform unbiased estimation of the system state vector. Therefore, the observer should include an integrator in order to overcome the problem of steady-state error. According to [18], one of the strategies to solve this problem consists in considering the increments of the input variables as the input of the model and the integral of these increments as a new state variable, yielding an extended model with an explicit integrator. This strategy is used in this paper. However, other approaches such as the one proposed in [19] may be used. This latter considers the use of a disturbance model in conjunction with an estimation of the disturbance (which is treated as state variable).

\section{RESUlTS AND Discussion}

The MPC controller has been tested in simulation for both reference tracking and disturbance rejection. A real implementation is an ongoing work developed by the authors. 


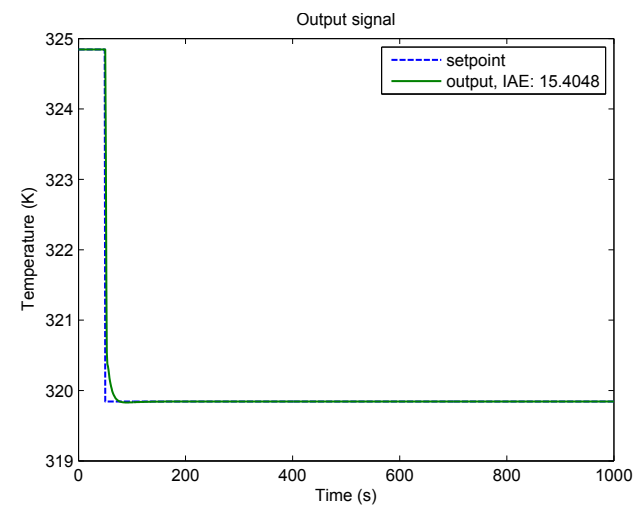

Fig. 6. Ideal response of the controlled system for reference tracking (same model for both controller and plant).

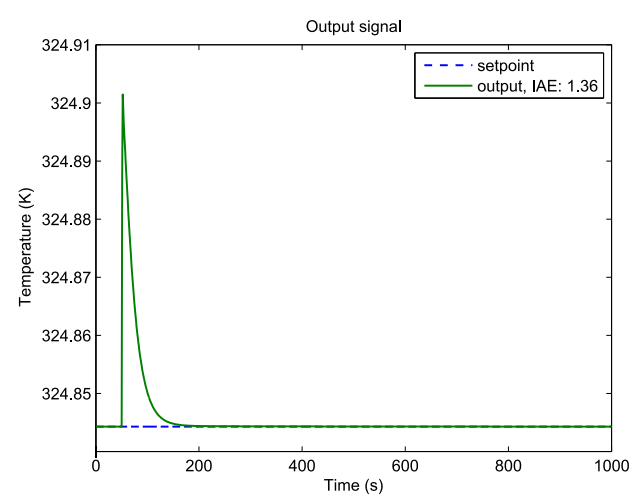

Fig. 7. Ideal response of the controlled system for disturbance rejection (same model for both controller and plant).

The benchmark plant corresponds to the full-order non-linear model presented in Section II. For the controller design, the reduced-order linear model (5) is used. In all cases, the Integral of the Absolute value of the Error (IAE) and the Total Control Input Variation (TCIV), i.e., the sum of the absolute value of the variation in the control signal for each sampling time, are chosen as performance indicators. In the reference tracking case, the system is required to reduce its output temperature by $5 \mathrm{~K}$. The disturbance is given by a rise in the stack power. This rising corresponds to an increment of $5 \mathrm{~A}(0.41 \mathrm{~V} /$ cell decrease in the unregulated voltage).

For comparison purposes only, the ideal response - when the plant and the controller have the same model (5) - is presented in Fig. 6 for the tracking case and in Fig. 7 for the disturbance rejection case. Figures show the proper performance for both reference tracking (IAE of 15.4) and disturbance rejection. For the latter case, the maximum deviation is about $0.05 \mathrm{~K}$, and the attenuation of the disturbance is done in less than $150 \mathrm{~s}$. For a the realistic case, where models for the controller and the plant are different ${ }^{4}$, the performance of the closed loop is shown in Figures from 8 to 11 . In the case of reference tracking, the IAE is 47 and a

${ }^{4}$ The plant corresponds with the non-linear model of Section II, while the MPC controller is based on model (5).

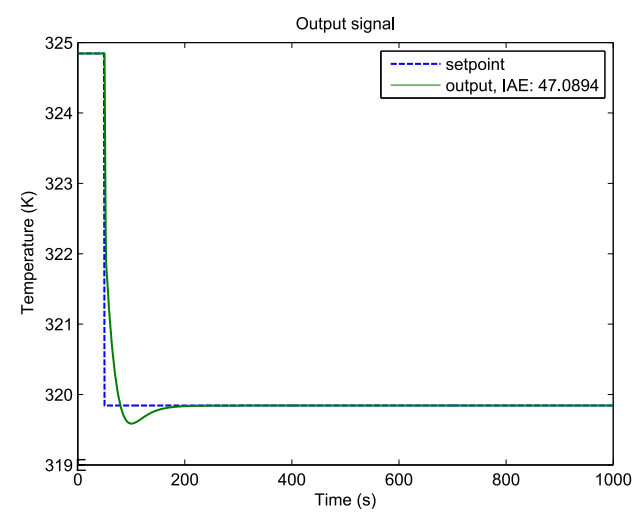

Fig. 8. Response of the controlled system for reference tracking.

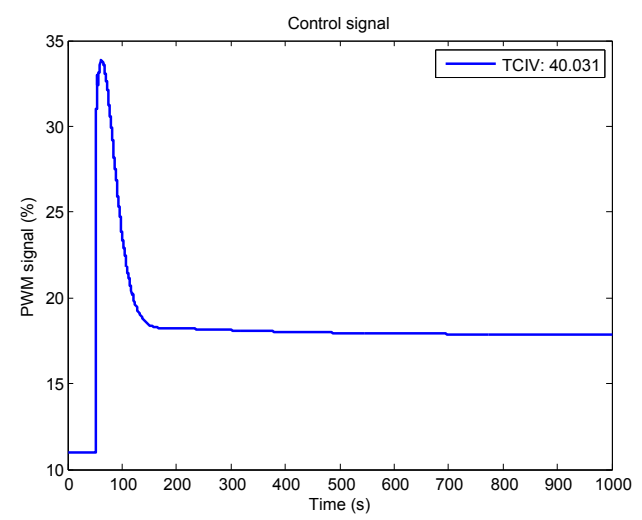

Fig. 9. Control signal for reference tracking.

small overshoot is presented. However, the controller is able to drive the temperature to its new reference value without steady-state error in less than $150 \mathrm{~s}$. In this case, the restriction over the change of the control signal is active (the controller is designed for a maximum control signal variation of $10 \%$ ). The corresponding TCIV is 40.0.

On the other hand, given that the observer is designed for steady-state offset-free estimation, the state variable and their estimate differ during the transitory state. In the regulatory case shown in Fig.10, this difference leads to a peak in the control signal that, in turn, induces the outlet-water temperature to fall. Due to this fact, the output response goes in opposite direction than in the ideal case shown in Fig.7. However, it is worth noticing that the maximum deviation in this case is around $1 \mathrm{~K}$, which is acceptable for this particular system. Moreover, the outlet-water temperature is driven towards a new reference value in around $150 \mathrm{~s}$ with a low TCIV (9.26), as shown in Fig. 11.

A PI controller has been also considered for comparison purposes. An analytical approach has been used to tune the parameter of this controller, using a first-order plant model, with an anti-wind-up circuit and considering using existing tools [20], [21]. The response of the closed-controlled system has been selected to have approximately the same settling time than with the MPC. The performance for reference tracking 


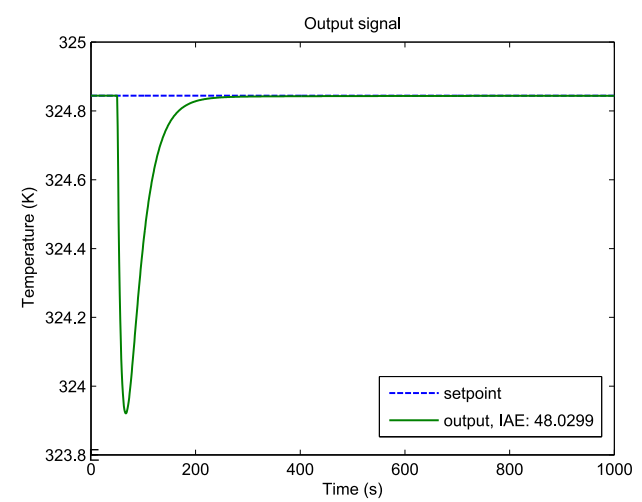

Fig. 10. Response of the controlled system for disturbance rejection.

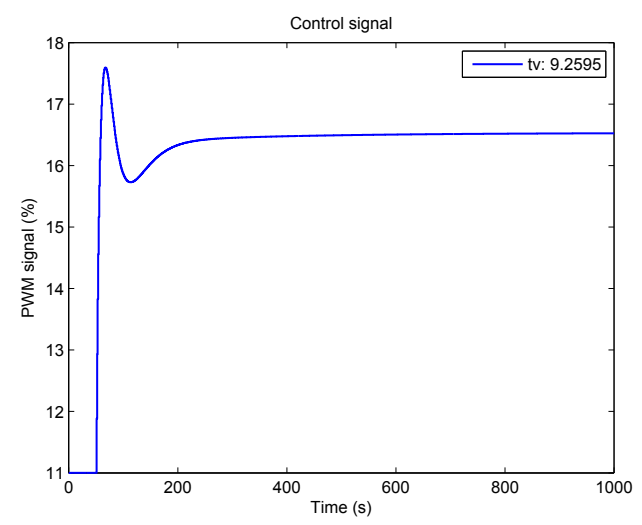

Fig. 11. Control signal for disturbance rejection.

is given in Fig. 12 and Fig. 13. As it can be seen, the output response is quite similar (in the IAE sense) as expected, since the PI controller has been tuned to accomplish this behaviour, but the TICV much higher. The slightly improvement in the IAE from the PI controller comes from this increment in the TCIV. Since the difference in the control signal is constrained by the MPC controller, a smoother control signal can be achieved. Regarding the disturbance rejection case shown in Fig. 14 and Fig. 15, the PI controller outperformed the MPC scheme with lower IAE and TCIV. However, it has to be taken into account that, using the PI controller, it not possible to add constraints as in the MPC case, which is the main reason to use this control methodology.

As said before, the system is required to comply with the temperature difference constraint (9) with $T_{\text {diff }}^{\max }=10 \mathrm{~K}$. In order to test the controller action when this constraint becomes active, in Fig. 16 the controlled system is tested with $T_{\text {diff }}^{\max }=4.5 \mathrm{~K}$. With this constraint active, the maximum difference between the inlet water temperature and the outlet water temperature is $4.27 \mathrm{~K}$. However, for the former case $\left(T_{\text {diff }}^{\max }=10 \mathrm{~K}\right)$, the temperature difference reaches $5.12 \mathrm{~K}$. As expected, a performance degradation occurred: the IAE value becomes 118.4 from the 47.1 in the no-constrained case.

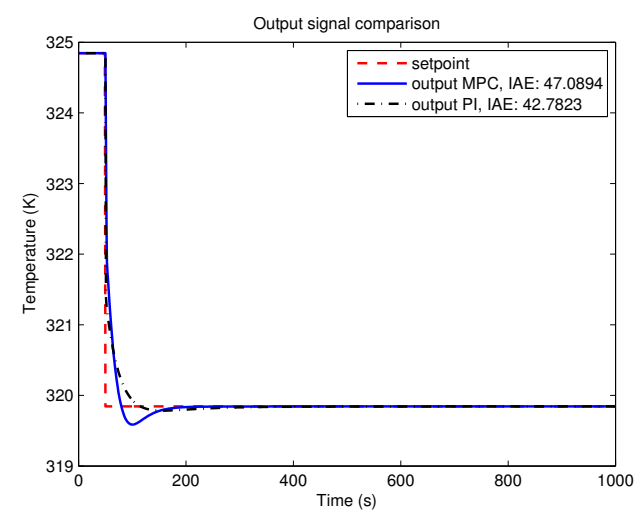

Fig. 12. Comparison of the MPC controller with a PI controller for servo response.

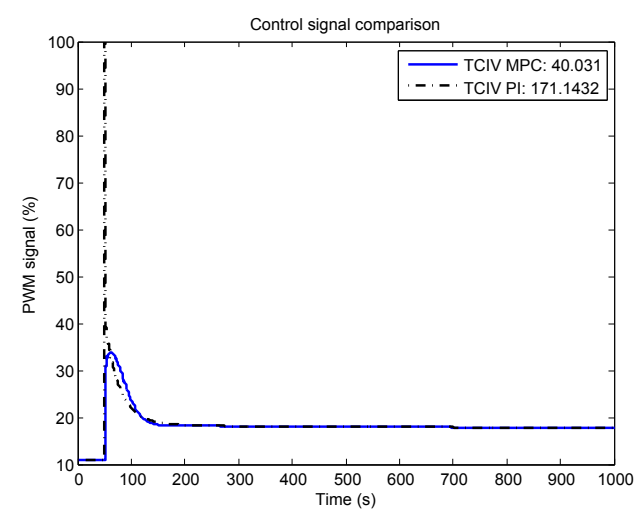

Fig. 13. Control signal of PI and MPC, for servo response.

\section{CONCLUSIONS}

In this paper, a thermal model for a 20-cell water-cooled PEM-FC stack was presented. The modelling procedure splits the stack in submodels that are concatenated in order to have the temperature dynamical behaviour along the stack. Also, a dynamical model of the heat exchanger is presented.

In order to control the outlet-water temperature of the stack, an MPC controller design is proposed, which takes into account the measurable disturbances and offers an offset-

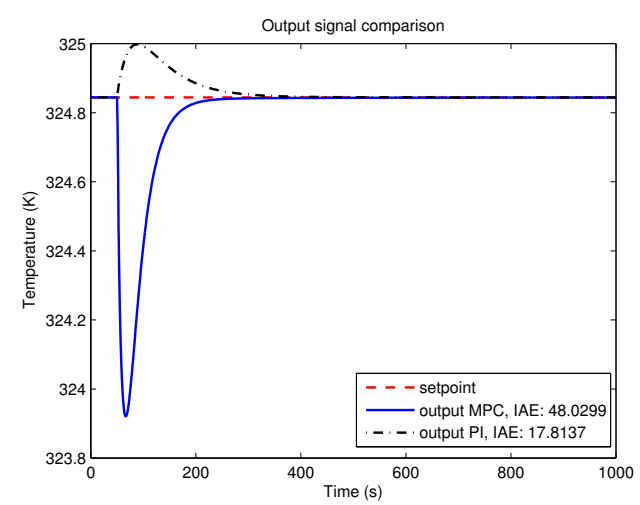

Fig. 14. Comparison of the MPC controller with a PI controller for disturbance rejection. 


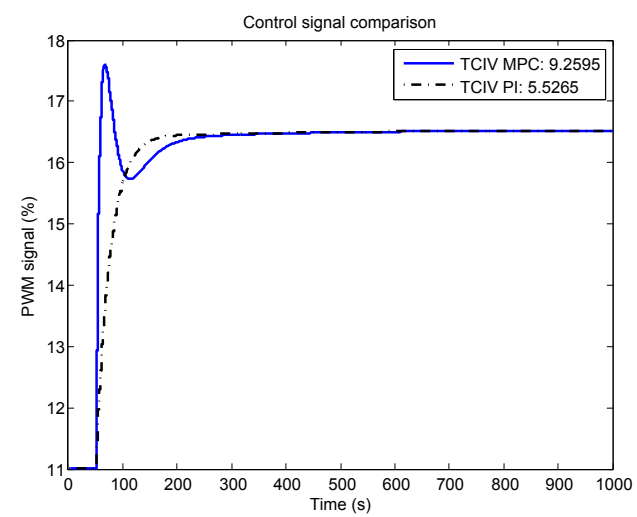

Fig. 15. Control signal of PI and MPC for disturbance rejection.

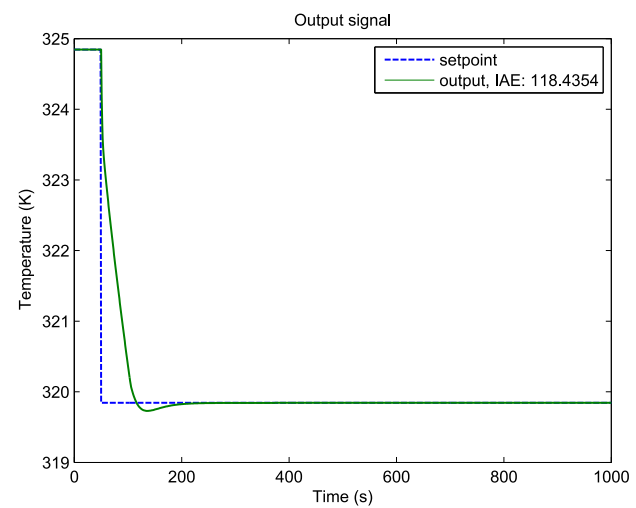

Fig. 16. Test where the temperature difference constraint is active

free response. The performance of the closed-loop controlled system was discussed based on simulations using the resultant non-linear model as virtual plant. The effectiveness of the approach for reference tracking and disturbance rejection was shown even considering the natural mismatch between the linear model used for control synthesis and the non-linear model.

Once the suitability of the approach has been confirmed, the following stage will be the development and implementation of the proposed control designs in the real water-cooled PEM-FC stack where the both models (of linear and non-linear nature) were validated.

\section{ACKNOWLEDGEMENT}

The research work of José David Rojas has been supported by the project 322-B2-727 of the Vicerrectoría de Investigación, Universidad de Costa Rica. The research work of C. Ocampo-Martinez has been supported by the project MACPERCON (Ref. 201250E027) of the Spanish National Research Council (CSIC). The research of C. Kunusch has been supported by the Seventh Framework Programme of the European Community through the Marie Curie actions (GA: PCIG09-GA-2011-293876), the Puma-Mind project (GA: FCH-JU-2011-1-303419), the CICYT project DPI201125649 (MINECO-Spain) and the CSIC JAE-DOC Research Programme.

\section{REFERENCES}

[1] F. Barbir, PEM Fuel Cells: Theory and Practice, 1st ed., ser. Sustainable World. Elsevier Academic Press, 2005.

[2] Y. Zhang, M. Ouyang, Q. Lu, J. Luo, and X. Li, "A model predicting performance of proton exchange membrane fuel cell stack thermal systems," Applied Thermal Engineering, vol. 24, no. 4, pp. 501 - 513, 2004.

[3] L. Dumercy, R. Glises, J. Kauffmann, and H. Louahlia-Galous, "Transient thermal computation of a pem fuel cell by a nodal modeling," in Vehicular Technology Conference, 2003. VTC 2003-Fall. 2003 IEEE 58 th, vol. 5, October 2003, pp. 3299 - 3303 Vol.5.

[4] X. Yu, B. Zhou, and A. Sobiesiak, "Water and thermal management for Ballard PEM fuel cell stack," Journal of Power Sources, vol. 147, no. 1-2, pp. $184-195,2005$.

[5] A. Salah, J. Gaber, R. Outbib, O. Serres, and H. El-Sayed, "Modeling and Simulation of PEM Fuel Cell Thermal Behavior on Parallel Computers," Energy Conversion, IEEE Transactions on, vol. 25, no. 3, pp. $768-777$, September 2010.

[6] J. Maciejowski, Predictive control with constraints. Pearson Education, 2002.

[7] A. Vahidi, A. Stefanopoulou, and H. Peng, "Current management in a hybrid fuel cell power system: A model-predictive control approach," Control Systems Technology, IEEE Transactions on, vol. 14, no. 6, pp. $1047-1057$, nov. 2006.

[8] C. Bordons, A. Arce, and A. del Real, "Constrained predictive control strategies for pem fuel cells," in American Control Conference, 2006, Minneapolis (USA), june 2006, p. 6 pp.

[9] A. Arce, D. Ramirez, A. del Real, and C. Bordons, "Constrained explicit predictive control strategies for pem fuel cell systems," in Decision and Control, 2007 46th IEEE Conference on, New Orleans, USA, December 2007, pp. $6088-6093$.

[10] M. Fiacchini, T. Alamo, C. Albea, and E. Camacho, "Adaptive model predictive control of the hybrid dynamics of a fuel cell system," in Control Applications, 2007. CCA 2007. IEEE International Conference on, Singapore, oct. 2007, pp. $1420-1425$

[11] Q. Chen, S. Quan, and C. Xie, "Nonlinear predictive control for oxygen supply of a fuel cell system," in Neural Networks, 2009. IJCNN 2009. International Joint Conference on, june 2009, pp. 518 -521.

[12] A. del Real, A. Arce, and C. Bordons, "Hybrid model predictive control of a two-generator power plant integrating photovoltaic panels and a fuel cell," in Decision and Control, 2007 46th IEEE Conference on, New Orleans, USA, dec. 2007, pp. $5447-5452$.

[13] M. A. Danzer, S. J. Wittmann, and E. P. Hofer, "Prevention of fuel cell starvation by model predictive control of pressure, excess ratio, and current," Journal of Power Sources, vol. 190, no. 1, pp. 86 - 91, 2009.

[14] J. Moré, P. Puleston, C. Kunusch, and A. Visintin, "Temperature control of a PEM fuel cell test bench for experimental MEA assessment," International Journal of Hydrogen Energy, vol. 35, no. 11, pp. 5985 - 5990, 2010.

[15] C. J. Geankoplis, Procesos de Transporte y Operaciones Unitarias, 3rd ed. Compañía Editorial Continental, S.A. DE C.V. México, 1998.

[16] B. Sohlberg, "Grey box modelling for model predictive control of a heating process," Journal of Process Control, vol. 13, no. 3, pp. $225-$ 238, 2003.

[17] K. Ogata, Discrete-time control systems, 2nd ed. Prentice Hall, Inc, 1995.

[18] A. González, E. Adam, and J. Marchetti, "Conditions for offset elimination in state space receding horizon controllers: A tutorial analysis," Chemical Engineering and Processing: Process Intensification, vol. 47, no. 12 , pp. $2184-2194,2008$.

[19] F. Borrelli and M. Morari, "Offset free model predictive control," in Decision and Control, 2007 46th IEEE Conference on, 2007, pp. 12451250 .

[20] K. Åström and B. Wittenmark, Computer Controlled Systems: Theory and Design, 3rd ed. Prentice Hall, 1996.

[21] K. Pirabakaran and V. Becerra, "Automatic tuning of PID controllers using model reference adaptive control techniques," in Proceedings of the 27th Annual Conference of the IEEE Industrial Electronics Society (IECON'01), 2001, pp. 736-740. 